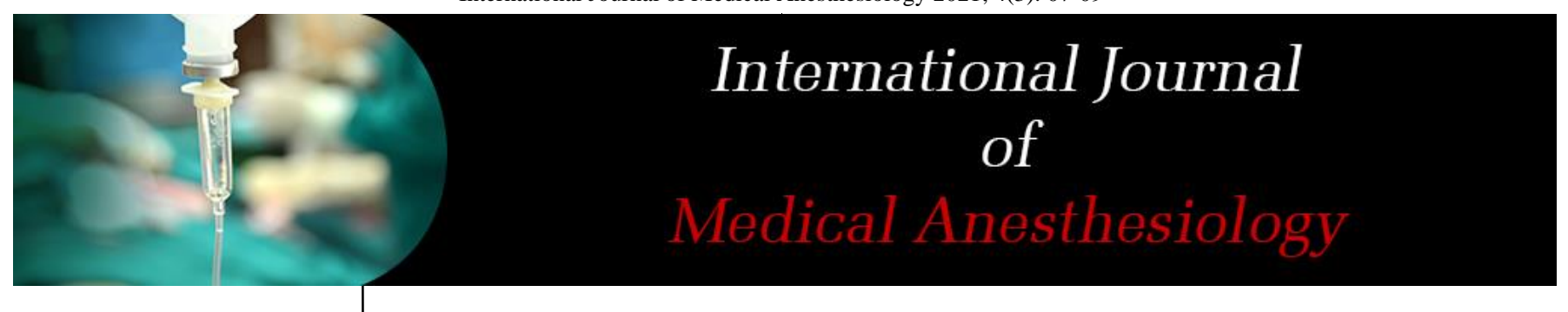

E-ISSN: 2664-3774

P-ISSN: 2664-3766

www.anesthesiologypaper.com

IJMA 2021; 4(3): 07-09

Received: 04-05-2021

Accepted: 06-06-2021

Dr. P Veena

Assistant Professor,

Department of

Anaesthesiology, Sree

Uthradom Thirunal Academy

of Medical Sciences,

Thiruvananthapuram, Kerala,

India

Dr. Girish Kumar J

Senior Resident, Department of Anaesthesiology, Sree

Uthradom Thirunal Academy of Medical Sciences,

Thiruvananthapuram, Kerala, India

Corresponding Author: Dr. P Veena

Assistant Professor,

Department of

Anaesthesiology, Sree

Uthradom Thirunal Academy

of Medical Sciences,

Thiruvananthapuram, Kerala,

India

\section{A case report on anaesthetic management of patient with bilateral retinitis pigmentosa with partial blindness for abdominal hysterectomy: Sequential combined spinal epidural anaesthesia in era of COVID-19 pandemic}

\section{Dr. P Veena and Dr. Girish Kumar J}

DOI: $\underline{\text { https://doi.org/10.33545/26643766.2021.v4.i3a.272 }}$

\section{Abstract}

Retinitis pigmentosa is typically bilateral and symmetric. There is currently no treatment that can stop the process of retinitis pigmentosa, but gene therapy shows promise. Retinitis pigmentosa is typically a bilateral, progressive retinal degeneration that ultimately leads to death of both rod and the cone photoreceptors. Retinitis pigmentosa is generally symmetric, but can present asymmetrically. Other complications associated with retinitis pigmentosa include posterior subcapsular cataracts and cystoid macular edema, retinal detachment ${ }^{[1]}$. Forty-five separate gene loci have been identified that account for $50 \%$ of all bilateral retinitis pigmentosa cases. In addition, between 20-30\% of patients with bilateral retinitis pigmentosa have an associated systemic disease, with more than 30 syndromes identified? The most widely known associated syndrome, Usher Syndrome, results in retinitis pigmentosa and hearing impairment. Other syndromes associated with retinitis pigmentosa are Laurence-Moon-Bardet-Biedl syndrome, Bardet-Biedl syndrome.

We report the case of Retinitis pigmentosa with partially blind patient for Total abdominal hysterectomy managed by Sequential combined Spinal Epidural Anaesthesia (Sequential CSEA) in the era of COVID Pandemic inorder to avoid drastic changes in blood pressure thus preventing drastic change in intraocular pressure as intraocular pressure is a determining factor in partially blind patient with progressive retinitis pigmentosa. Sequential combined spinal epidural anaesthesia is a very safe regional anaesthesia technique to avoid General anaesthesia thus preventing abrupt increase in intraocular pressure and aerosol generation in era of COVID Pandemic. Sequential combined spinal epidural anaesthesia (Sequential CSEA) is probably the greatest advance in central neuraxial block. Sequential combined spinal epidural anaesthesia is a safe, effective, reliable technique with stable haemodynamic along with provision of prolonging analgesia compared to spinal anaesthesia alone for high risk patients.

Keywords: retinitis pigmentosa, sequential combined spinal epidural anaesthesia, retinal detachment, bupivacaine, postoperative pain

\section{Introduction}

The true etiology of retinitis pigmentosa is difficult to determine due to the small number of cases. Sporadic appearance of the disease is more likely than genetic manifestation in both unilateral and bilateral retinitis pigmentosa. Decrease of visual acuity occurs mainly in later stages of the disease, especially if cystoid macular oedema and subcapsular cataract develop. RP can occur sporadically or hereditary. The inheritance pattern can be autosomal dominant, autosomal recessive or X-chromosomal. Because of the progressive nature of this genetic disorder visual acuity progressively decrease with years even leading to retinal detachment with drastic changes in intraocular pressure.

Considering the patient's present condition of partial blindness and prevailing pandemic condition we decided to avoid general anaesthesia as far as possible to avoid drastic changes in intraocular pressure, aerosol generation ${ }^{[1,2]}$.

Combined spinal epidural, single segment, needle though needle technique is gaining popularity in modern anaesthesia practice. It offers rapid onset, efficacy and safety with minimal chances of toxic effects combined with potential for improving an inadequate block and prolonging duration of analgesia Intraoperatively and post operatively. 
This technique reduces or eliminates some of the disadvantages of spinal anaesthesia while preserving their advantages.

A more improved method called the modified combined spinal epidural technique or the sequential combined spinalepidural technique, in which a spinal dose intended to be inadequate for surgery is used in an attempt to reduce hypotension and the block is then deliberately extended cephalad with the epidural drug. This technique is becoming increasingly popular in modern obstetric practice, because of various claimed benefits, mainly stable haemodynamic status ${ }^{[3]}$. The sequential CSEA must be used in elderly high risk patients for surgery with encouraging results.

\section{Case report}

We are reporting the case of 50 year old nullipara with long duration bilateral Retinitis pigmentosa partially blind with associated anemia posted for total abdominal hysterectomy in view of ongoing bleeding per vaginum inspite of conservative medical management including blood transfusion.

No past history of surgeries or anaesthesia exposure. No Family history of similar genetic disorder.

\section{On physical examination}

Weight $45 \mathrm{~kg}$, BMI of 21, Pallor present, Conscious with BP $-130 / 80 \mathrm{mmg}$,

HR-88/min, regular, normal rhythm, high volume.

Ophthalmic examination revealed - poor light fixation both eyes, Nystagmus, bilateral pupils sluggish reaction to light, Intraocular pressure - normal

Bilateral Fundoscopic examination - disc pale (waxy), arteriolar narrowing, bone corpuscles like pigments over fundus.

Intravenous access as well as regional anaesthesia access difficultly was anticipated. Other systemic examination reveals no abnormality.

Airway Assessment after COVID RTPCR - Revealed mallampatti class 2

Spine examination - Painless and non-tender revealed a very narrow interspinous space without kyphoscoliosis, no neurological deficits. Regional anaesthesia access difficultly was anticipated

Investigations- Hemoglobin $9.6 \mathrm{~g} / \mathrm{dl}$ other investigations were within normal

Electrocardiography \& Chest xray - Normal

\section{Anaesthetic management}

After arranging adequate blood products, a detailed written informed high risk consent (in view of retinitis pigmentosa and its progressive nature) was obtained for the planned anaesthesia procedure of combined sequential spinal epidural anaesthesia along with a backup plan for general anaesthesia in view of anticipated difficult regional anaesthesia access.

Sedative premedication was given on preop night, Patient was reassured to ally anxiety and was given T. Pantoprazole $40 \mathrm{mg}$, T. Alprazolam $0.25 \mathrm{mg}$ morning of surgery. N.P.O was maintained.

After preparation of OT, checking anaesthesia machine with emergency resuscitation drugs, intravenous fluids, infusion pump, difficult regional anaesthesia equipments and equipment backup for general anaesthesia patient was shifted to OT.
On receiving inside OT: Monitors for $\mathrm{ECG}$, Non-invasive Blood pressure, pulse oximetry, temperature probe were connected. Two wide bore cannulae were established under strict asepsis under local anaesthesia and was preloaded with balanced salt solution.

In view of anticipated difficult regional anaesthesia access sitting position was opted for Sequential Combined Spinal Epidural anaesthesia.

Under strict asepsis under local anaesthesia with $2 \%$ plain lignocaine, L3-L4 Space was infilterated for epidural needle placement. In L3-L4 space, $18 \mathrm{G}$ Tuohy needle was inserted, epidural space was identified by loss of resistance to air technique as well as by Hanging drop technique. Epidural catheter was threaded and fixed at $10 \mathrm{~cm}$ from skin, then on lifting up the epidural catheter a fall in liquid meniscus level in the epidural catheter further confirmed epidural space. Following which a test dose of $2 \mathrm{ml} 2 \%$ lignocaine with 1 in 2lakh adrenaline was given which revealed negative for intravascular/intrathecal route.

Under strict asepsis, in sitting position, under local anaesthesia was given in L4L5 space, 23 G Quincke spinal needle was inserted, following which a clear CSF flow was obtained and $0.5 \%$ heavy Bupivacaine $2 \mathrm{ml}$ was given. Sensory blockade was assessed in supine position After which $1.5-2 \mathrm{ml}$ of $0.5 \%$ isobaric bupivacaine was given for every unblocked segment through epidural route to extend block to T6 (block was assessed in supine position).

Motor block of lower limbs was assessed bilaterally using Bromage Scale (grade I inability to move feet, II able to move feet only, III just able to move knees, IV full flexion of knee and feet).

Intra-operatively Epidural top up with $3 \mathrm{ml} 2 \%$ Lignocaine with adrenaline and $3 \mathrm{ml}$ of $0.5 \%$ bupivacaine given followed by epidural infusion with $0.5 \%$ bupivacaine at $4 \mathrm{ml} / \mathrm{hr}$ with urine output monitoring. Intraoperative blood loss was managed with judicious crystalloids and blood. Surgery completed uneventful.

Postoperative analgesia was with epidural anaesthesia with $0.2 \%$ ropivacaine at 3 to $4 \mathrm{ml} /$ hour and supplemented with Epidural buprenorphine at 180 microgram at twelve hourly interval.

\section{Discussion}

Sequential combined spinal epidural anaesthesia (Sequential CSEA) is probably the greatest advance in central neuraxial block. Sequential combined spinal epidural anaesthesia is a safe, effective, reliable technique with stable haemodynamic along with provision of prolonging analgesia compared to spinal anaesthesia alone for high risk geriatric patients undergoing major orthopaedic surgery ${ }^{[4]}$. Studies reveal that spinal anaesthesia combined with epidural technique has been shown to ameliorate the hyperglycemic as well as catecholamine surge to surgery. Epidural regional anesthesia is safe and cost-effective technique for providing quality surgical anesthesia and prolong post-operative pain relief and thus also effective in blunting autonomic, somatic and endocrine response triggered by surgical insult 5 . International consensus on neuroaxial anesthesia and analgesia revised and accepted for use of a poly pharmacological approach for treatment of intra and postoperative pain and relaxation. The effect of epidural anesthesia and analgesia on cardiovascular coagulation, pulmonary, gastrointestinal physiology, surgical stress response, immune function cognition, complications and 
surgical outcomes are studied extensively and results are excellent in favor of epidural anesthesia. In this patient epidural anesthesia avoided intraocular changes, cardiac depressant general anesthetics drugs, nephrotoxic drugs respiratory depressant drugs etc. which made the operative goal achieved. To reduce the incidence and severity of hypotension a sequential combined spinal epidural technique has been described in obstetric practice in which a spinal dose of local anaesthetic intended to be inadequate for surgery is used in an attempt to reduce hypotension. The block is then deliberately extended cephalad with the epidural drug. The onset of block is not delayed by this method but at the same time adequate level of sensory block is obtained. The sequential CSEA is particularly advantageous in high risk patients where gentler onset of sympathetic block is desirable to reduce haemodynamic side effects. The block in sequential CSEA resulted from a relatively small amount of the local anaesthetic through spinal route followed by epidural drug which help to increase the subarachnoid block to desired level which conforms to the study of Swami et al. for caesarean section. Many considerations have been given as to how epidural top up works after a spinal anaesthesia in sequential CSEA ${ }^{[6]}$.

1) Continuing spread of initial subarachnoid block (correlated to epidural injection).

2) Existence of sub clinical analgesia at a higher level, which is enhanced and becomes evident by perineural transdural spread of epidural local anaesthetic.

3) Leakage of epidural local anaesthetic though the dural hole in subarachnoid space.

4) Change in epidural pressure. The pressure becomes atmospheric which may result in better spread of local anaesthetic through an effect on volume and circulation of CSF.

5) Compression of the theca by the epidurally injected volume of local anaesthetic (or even saline) solution resulting in a 'squeezing' of CSF and more extensive spread of spinal local anaesthetic.

6) Combination of last two mechanisms.

\section{Acknowledgement}

Authors would like to thank Department of Gynaecology and Department of Anaesthesiology, All anaesthesiology technicians and Operation theatre nursing staffs Postoperative ICU staffs.

\section{References}

1. Chan WO, Brennan N, Webster AR, Michaelides M, Muqit MMK. Retinal detachment in retinitis pigmentosa. BMJ Open Ophthalmol 2020;5(1):e000454. doi: 10.1136/bmjophth-2020000454. PMID: 3267

2. Cousins MJ. The spinal route of analgesia. Acta Anaesthesiol Belg Suppl 2 1988;39:71-82

3. Dureja GP, Madan R, Kaul HL. Combined spinal epidural anaesthesia. In : Regional anaesthesia and pain management (current perspectives) B. I. Churchill Livingstone Pvt. Ltd 2000, 139-45

4. Dr. P Veena, Dr. Narayanan Unnithan H, Dr. Divya V, Dr. Jeetish Mathew. Graded epidural anaesthesia as the sole anaesthetic technique for a life salvaging below knee amputation on a high risk patient: A case report. Int J Med Anesthesiology 2021;4(2):29-31. DOI: 10.33545/26643766.2021.v4.i2a.230
5. Holmstrom E, Laugaland K, Rawal N, Haliberg S. combined spinal epidural block versus spinal and epidural block for orthopaedic surgery. Can J Anaesth 1993;10(7):601-06.

6. https://www.researchgate.net/publication/351250180_T aylor's_ApproachModified_paramedian_approach_a_g ame_changer_in_unanticipated_difficult_spine_during the_era_of_Covid_pandemic_-_A_Case_Report

7. Dr P Veena, "The Role of Perioperative Physicians with Nerves of Steel during SARS COV 19 pandemic unwinding few experience as a COVID 19 warrior.", IJRAR - International Journal of Research and Analytical Reviews (IJRAR), E-ISSN 2348-1269, PISSN 2349-5138, 2021;8(2):923-925 Available at : http://www.ijrar.org/IJRAR21B1116.pdf 